SHORT REPORT

\title{
Diffuse brain oedema in idiopathic intracranial hypertension: a quantitative magnetic resonance imaging study
}

\author{
M E Bastin, S Sinha, A J Farrall, J M Wardlaw, I R Whittle
}

See end of article for

authors' affiliations

J Neurol Neurosurg Psychiatry 2003;74:1693-1696

....................

Correspondence to:

Dr Mark Bastin, Medical

and Radiological Sciences

(Medical Physics),

University of Edinburgh,

Western General Hospital,

Crewe Road, Edinburgh

EH4 2XU, UK; meb@

skull.dcn.ed.ac.uk

Received 27 March 2003

In revised form

20 May 2003

Accepted 24 May 2003

\begin{abstract}
Objectives: To investigate the hypothesis that idiopathic intracranial hypertension is associated with diffuse brain oedema, using quantitative magnetic resonance imaging.

Methods: Values for the mean diffusivity of water $(\langle\mathrm{D}\rangle)$ and the proton longitudinal relaxation time (T1) were measured for various brain regions in 10 patients with idiopathic intracranial hypertension and 10 age, sex, and weight matched controls.

Results: No significant differences in $\angle D>$ and $T 1$ values were found between patient and control groups in any of the brain regions investigated.

Conclusions: The results suggest that idiopathic intracranial hypertension is not associated with abnormalities of convective transependymal water flow leading to diffuse brain oedema.
\end{abstract}

.....................

diopathic intracranial hypertension (IIH), also known as pseudotumour cerebri, is a chronic disorder of unknown aetiology which is characterised by raised intracranial pressure. The features of this condition-which typically affects young obese women-are headache, visual disturbance, and papilloedema in the absence of abnormalities on structural magnetic resonance (MR) imaging and in cerebrospinal fluid (CSF) composition. ${ }^{1-4}$ Potential mechanisms underlying IIH include brain swelling caused by diffuse brain oedema, increased sagittal sinus venous pressure, increased cerebral blood volume, increased rate of CSF production, and disturbance of CSF circulation. ${ }^{56}$

The presence of diffuse brain oedema and its role in the raised intracranial pressure associated with IIH can be assessed non-invasively using MR imaging techniques that characterise the properties of brain water. Two such methods are diffusion weighted MR imaging (DWMRI) and quantitative mapping of the proton longitudinal relaxation time (T1). In the former technique, the mean diffusivity $(<\mathrm{D}>)$, which reflects the average microscopic mobility of brain water molecules, can be determined from an MR imaging experiment in which diffusion sensitising gradients are applied in three orthogonal directions. ${ }^{7}$ Quantitative maps of $\mathrm{Tl}$ provide useful information on the spatial distribution of brain water content. $^{8}$

In this paper we report results from a pilot study in which MR imaging was used to measure values of $\langle\mathrm{D}\rangle$ and $\mathrm{Tl}$ for various different brain regions in 10 patients with IIH and in a control group of age, sex, and weight matched volunteers. By directly comparing these quantitative $\mathrm{MR}$ imaging parameters in the two subject groups it is possible not only to investigate the hypothesis that IIH is caused by diffuse oedema and brain swelling, but also to identify which regions, if any, are affected by changes in brain water homeostasis.

\section{METHODS}

\section{Subjects}

Ten female patients with a clinical diagnosis of IIH and no contraindications to MR were recruited consecutively from neurology and ophthalmology clinics and enrolled in the study. The diagnostic criteria for IIH were fulfilled in all cases; these are:

- increased mean intracranial pressure measured by lumbar puncture, and greater than $20 \mathrm{~cm} \mathrm{H}_{2} \mathrm{O}$;

- no evidence of focal or diffuse disease from the radiological data;

- no evidence of major venous sinus thrombosis on MR venography;

- normal protein concentration and normal cell count in the CSF.

All patients were symptomatic, with headache or visual disturbances or both. Two patients had lumboperitoneal shunts but these were confirmed on clinical and lumbar puncture examination to be non-functional. None of the patients was undergoing corticosteroid treatment. Ten healthy women matched for age and weight, and with no history of neurological disease, were also recruited as controls.

The local ethics committee approved the study and informed consent was obtained from each subject.

\section{MR imaging acquisition parameters}

All MR imaging data were obtained using a GE Signa LX 1.5 T clinical scanner (General Electric, Milwaukee, Wisconsin,

Abbreviations: $A D C$, apparent diffusion coefficient; DW-MRI, diffusion weighted magnetic resonance imaging; $E P$, echo planar; $\mathrm{IH}$, idiopathic intracranial hypertension; IR, inversion recovery; ROI, region of interest; $\mathrm{Tl}$, inversion time 
USA) and manufacturer-supplied "birdcage" quadrature head coil. Each patient underwent MR imaging, which consisted of a standard fast spin echo T2 weighted sequence, DW-MRI and Tl mapping protocols. The duration of the examination was approximately 20 minutes.

In the DW-MRI experiment, DW images were acquired using a single shot, spin echo, echo planar (EP) imaging sequence in which two symmetric trapezoidal gradient pulses of duration $\delta=32.2 \mathrm{~ms}$, separation $\Delta=39.1 \mathrm{~ms}$, and rise time $\varepsilon=1.2 \mathrm{~ms}$ were inserted around the $180^{\circ}$ refocusing pulse in the required gradient channel. Sets of axial DW-EP images $\left(b=0\right.$ and $1000 \mathrm{~s} / \mathrm{mm}^{2}$ ) were collected with diffusion gradients applied sequentially along the superior/inferior, anterior/posterior, and right/left directions. Two acquisitions consisting of a baseline T2 weighted EP image and three DWEP images, a total of eight images, were collected per slice position. The acquisition parameters for the DW-EP imaging sequence were 15 axial slices of $5 \mathrm{~mm}$ thickness and $1 \mathrm{~mm}$ slice gap, a field of view of $240 \times 240 \mathrm{~mm}$, an acquisition matrix of $128 \times 128$ (zero filled to $256 \times 256$ ), a TR (time of repetition) of $10 \mathrm{~s}$, and a TE (time of echo) of $98.8 \mathrm{~ms}$.

Maps of $\mathrm{Tl}$ values were obtained from 15 axial slices coincident with those imaged in the DW-MRI experiment using an inversion recovery (IR) single shot spin echo EP imaging sequence. To provide accurate values of $\mathrm{Tl}$, nine different inversion times (TI) were chosen-namely 50, 220, $760,1300,1840,2380,2920,3460$, and $4000 \mathrm{~ms}^{9}$ The remaining acquisition parameters for this IR-EP sequence were identical to those used in the DW-MRI experiment, except for a TR of $60 \mathrm{~s}$.

\section{Image analysis}

In the DW-MRI experiment, the set of two component EP images for each gradient direction was averaged to give four (one T2 weighted and three DW-EP images) high signal to noise ratio images for each slice. Within each voxel the three orthogonal apparent diffusion coefficient (ADC) components $\left(\mathrm{ADC}_{\mathrm{x}}, \mathrm{ADC}_{\mathrm{y}}\right.$, and $\left.\mathrm{ADC}_{\mathrm{z}}\right)$ were estimated by linear regression from the measured signal intensities in the EP images. To avoid diffusion anisotropy effects, ${ }^{7}$ the three orthogonal ADC components were averaged to produce $<\mathrm{D}>$.

The value of $\mathrm{Tl}$, the cosine of the inversion pulse flip angle $(\kappa)$, and the equilibrium signal intensity $\left(S_{0}\right)$ were determined for every voxel from the set of nine IR-EP images collected in each of the 15 slice locations using the equation ${ }^{9-11}: S\left(S_{0}, \kappa, T_{1}\right)=S_{0}\left[1-(1-\kappa) e^{-(T I / T 1)}\right]$

\section{Region of interest and statistical analysis}

To determine whether there were significant differences in brain water diffusion and proton longitudinal relaxation time parameters between the patient and control groups, a region of interest (ROI) analysis, similar to that employed by Gideon et $a{ }^{12}$ was undertaken for various grey and white matter structures-specifically, cortical grey matter, thalamus, head of the caudate nucleus, putamen, corpus callosum, posterior limb of the internal capsule, subcortical white matter, and centrum semiovale. Values of $\langle\mathrm{D}\rangle$ and $\mathrm{Tl}$ were obtained from multiple small circular (25 voxels, volume $110 \mathrm{~mm}^{3}$ ) ROI placed bilaterally in these brain structures (fig 1A). Partial volume effects were minimised by siting the ROI at least 3 voxels away from the edge of the ventricles and sulci. So that the observer was not influenced by values of the diffusion and relaxation time parameters, all ROI were defined on the T2 weighted EP images. As the T2 weighted EP images and the $<\mathrm{D}>$ and $\mathrm{Tl}$ parametric maps were by definition coregistered, this allowed these quantitative parameters to be measured simultaneously in each ROI. The observer (AJF) was blind to the clinical status of each subject and the purpose of the study.
For the patient and control groups, descriptive statistics were determined for the $\langle\mathrm{D}\rangle$ and $\mathrm{Tl}$ parameters in each ROI from the combined measurements made from each population. Statistical differences in these parameters between the two groups were assessed using an independent samples two tailed Student $t$ test (null hypothesis, $<\mathrm{D}>$ (patient) $-<\mathrm{D}>($ control $)=0$, etc $)$. In all cases a probability (p) value of $<0.05$ was considered statistically significant.

\section{RESULTS}

The age and weight ranges for the patient group were 16 to 49 years (mean (SD), 28.0 (10.0) years) and 63.5 to $127.0 \mathrm{~kg}$ $(92.2(23.9) \mathrm{kg})$. The mean duration of symptoms was 31.5 (22.7) months. The age and weight ranges for the controls were 18 to 41 years $(29.0$ (7.4) years) and 71.0 to $112.0 \mathrm{~kg}$ $(92.6(14.6) \mathrm{kg})$. There was no significant difference between the mean age and weight of the patient and control groups.

Table 1 shows values of $<\mathrm{D}>$ and $\mathrm{Tl}$ for the patient and control groups in each of the brain regions investigated. In these data there is no evidence for an increase in brain water diffusion and proton longitudinal relaxation time values in the patient group compared with the controls. In fact there was no statistically significant difference in either parameter in any region between the two subject groups. Also shown for comparison are published values of these parameters obtained from previous studies in normal volunteers. In all the regions investigated there is close agreement between the values of $\langle\mathrm{D}>$ and $\mathrm{Tl}$ measured in the current study and those reported previously. ${ }^{10}{ }^{13} 14$

\section{DISCUSSION}

There have been several recent reviews of the mechanisms underlying IIH. ${ }^{5}{ }^{6}$ Despite extensive studies of these mechanisms the relative contributions of each still remain unclear. In this paper the possible role of diffuse brain oedema in increasing cerebral volume was investigated by comparing brain water diffusion and proton longitudinal relaxation time values in patient and control groups.

There have been three previous studies of brain water diffusion parameters in IIH. Sorensen et al found evidence for increased diffusion in various brain regions in seven patients with IIH when compared with measurements made in a group of healthy controls. ${ }^{15}$ Specifically, increased water diffusion was observed in periventricular regions in three patients, and in the whole brain in the remaining four. These general results were replicated in a larger study of 10 patients and controls. ${ }^{16}$ It was argued that these data indicated that $\mathrm{IIH}$ is associated with abnormalities of convective transependymal water flow leading to diffuse brain oedema. Such a hypothesis is, however, not conclusively supported by the data obtained by Gideon et al in their study of 12 patients and 12 controls, ${ }^{12}$ where significantly increased water diffusion values were found only in subcortical white matter and not in other parenchymal regions. Nevertheless, in these three studies, IIH patients were found to have significantly higher $\mathrm{ADC}$ values than controls in some brain regions-a difference that was not observed in the current study.

The reason for this apparent discrepancy could arise from two main sources, namely differences in the subject populations and in the MR imaging protocols. Gideon et al, ${ }^{12}$ for example, imaged seven women and five men with IIH of mean age 38.6 (16.7) years and mean symptom duration of 22.3 (25.9) months. Although the mean age of our patient sample was younger (at $28.0(10.0)$ years) with longer symptom duration (31.5 (22.7) months), none of these differences is statistically significant. Furthermore, sex differences in the patient populations can also be discounted, as three of the seven women scanned by Gideon et al had 

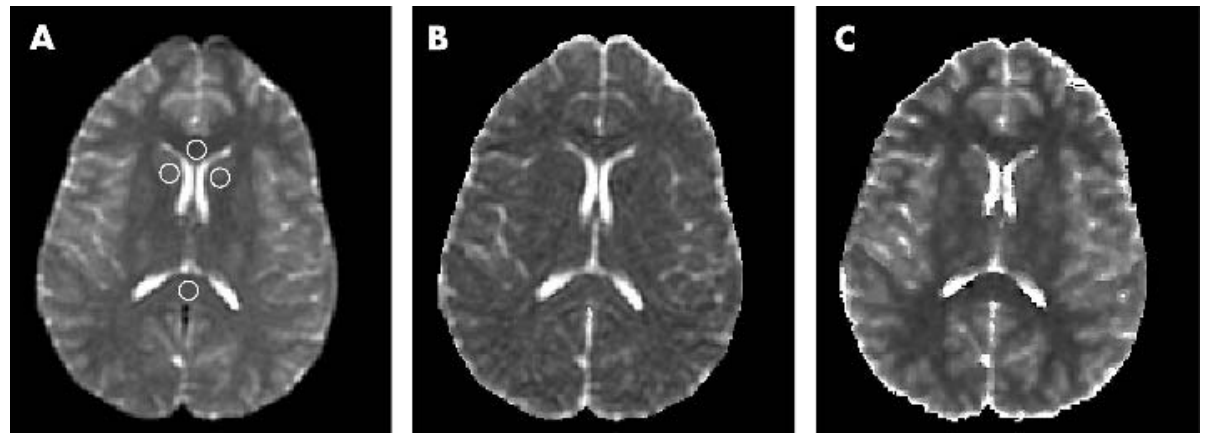

Figure 1 (A) T2 weighted echo planar (EP) image. (B) Mean diffusivity $(<D>$ ) and (C) proton longitudinal relaxation (T1) parametric maps obtained from a 19 year old female patient. The small circular regions of interest used to measure water diffusion and proton relaxation time parameters in the various brain regions (in this case head of the caudate nucleus and corpus callosum) are overlaid on the T2 weighted EP image.

raised ADC values in subcortical white matter. It should be noted, however, that the controls recruited by Gideon were not weight matched to the patients as in the current study. It is therefore unclear whether differences in the subject populations are really responsible for the contradictory results of these studies.

The difference between the MR imaging protocols employed in these studies is, however, much more significant. Specifically, in the three studies done by Sorensen et al 1516 and Gideon et al, ${ }^{12}$ diffusion parameters were measured using spin echo sequences with diffusion gradients applied in a single direction. While spin echo sequences can provide accurate ADC maps, any bulk patient motion will introduce significant artefacts into the component DW images. More importantly, it is now well recognised that the accurate measurement of the magnitude of water diffusion in vivo requires that the effects of diffusion anisotropy are removed. ${ }^{71}$ This is typically achieved by averaging the ADC values obtained from an MR imaging experiment in which diffusion sensitising gradients are sequentially applied in three orthogonal directions. By applying diffusion gradients in only one direction, it is unclear whether any observed differences between subjects reflect pathological changes or merely biological variation in fibre tract orientation and subject positioning in the magnet. In the current study the use of single shot EPI significantly reduces bulk patient motion, while $\langle\mathrm{D}\rangle$ is calculated from ADC components measured in three orthogonal directions.

Our study was undertaken to investigate whether there were gross differences in brain water diffusion parameters and proton longitudinal relaxation time values between IIH patients and suitably matched controls. Table 1 shows that in our sample population no such gross differences exist. Furthermore, the similarity between the values of $<\mathrm{D}>$ and $\mathrm{Tl}$ displayed in table $\mathrm{l}$ suggests that to detect a significant difference at this level would require a very large sample size. For example, a study with $80 \%$ power to detect a significant difference $(\mathrm{p}<0.05)$ in $<\mathrm{D}>$ values of subcortical white matter between patient and control groups would require 1014 subjects. However, although the methodology of the current study is more refined than that of Sorensen et al and Gideon et al, it is also possible that heterogeneity in the pathogenesis of IIH is responsible for the contradictory results of these studies. ${ }^{6}$

\section{Conclusions}

In this pilot study, brain water diffusion and proton longitudinal relaxation time parameters were measured in a small cohort of patients with idiopathic intracranial hypertension and controls to determine whether increased brain water was associated with this condition. No significant difference was found between these MR parameters in the two groups, which suggests that diffuse brain oedema is not a feature of IIH.

Table 1 Mean diffusivity $(\angle D>$ ) and proton longitudinal relaxation time (T1) measured in various brain regions for 10 female patients with idiopathic intracranial hypertension and 10 age, sex, and weight matched controls

\begin{tabular}{|c|c|c|c|c|c|c|c|c|}
\hline \multirow[b]{2}{*}{ Brain region } & \multicolumn{4}{|c|}{$<$ D $>\left(\times 10^{-6} \mathrm{~mm}^{2} / \mathrm{s}\right)$} & \multicolumn{4}{|l|}{$\mathrm{Tl}$ (ms) } \\
\hline & Patient & Control & $p$ Value & $\begin{array}{l}\text { Published } \\
\text { values }^{a}\end{array}$ & Patient & Control & p Value & Published values ${ }^{c}$ \\
\hline \multicolumn{9}{|l|}{ Grey matter } \\
\hline Cortical grey matter & $842(124)$ & $816(92)$ & 0.28 & $930(20)$ & $1024(146)$ & $1070(221)$ & 0.27 & $1113(48)$ \\
\hline Thalamus & $738(30)$ & 719 (38) & 0.20 & $730(50)$ & $852(60)$ & $835(44)$ & 0.29 & $814(26)$ \\
\hline Caudate (head) & $764(34)$ & $771(45)$ & 0.62 & $680(70)$ & 1075 (71) & $1103(73)$ & 0.23 & $1033(41)$ \\
\hline Putamen & 727 (30) & 708 (38) & 0.20 & $720(90)$ & 1059 (96) & $1040(80)$ & 0.49 & 919 (42) \\
\hline \multicolumn{9}{|l|}{ White matter } \\
\hline Corpus callosum & $818(85)$ & $824(102)$ & 0.84 & $815(117)$ & $880(179)$ & $812(120)$ & 0.16 & $756(81)$ \\
\hline Internal capsule & 772 (65) & 762 (33) & 0.51 & $690(30)$ & 834 (133) & $848(94)$ & 0.71 & - \\
\hline Subcortical WM & 720 (44) & 726 (52) & 0.58 & 770 (30) & 748 (72) & 733 (43) & 0.27 & $642(21)$ \\
\hline Centrum semiovale & $678(52)$ & $662(65)$ & 0.42 & $(648(24))^{b}$ & $729(46)$ & $742(30)$ & 0.31 & - \\
\hline $\begin{array}{l}\text { Values are mean } \text { (SD } \\
\text { a Values }<D>\text { report } \\
\text { bValues of }<D>\text { rep } \\
\text { Values of } T 1 \text { reporte }\end{array}$ & $\begin{array}{l}\text { y Ulug ar } \\
\text { d by Pierp } \\
\text { y Kingsley }\end{array}$ & $\begin{array}{l}\text { n Zijl in } f \\
\text { et al in ei } \\
l \text { in three }\end{array}$ & $\begin{array}{l}\text { rmal vol } \\
\text { rmal vo } \\
\text { al volunt }\end{array}$ & s. $^{14} 1.5 \mathrm{~T}^{10}$ & & & & \\
\hline
\end{tabular}




\section{ACKNOWLEDGEMENTS}

This work was funded by the Cunningham Trust and undertaken at the SHEFC Brain Imaging Research Centre for Scotland (http:// www.dcn.ed.ac.uk/bic). Statistical advice was provided by Cat Graham of the Wellcome Trust Clinical Research Facility, Edinburgh (http://www.wtcrf.ed.ac.uk).

\section{Authors' affiliations}

M E Bastin, Medical and Radiological Sciences (Medical Physics), University of Edinburgh, Edinburgh, UK

S Sinha, A J Farrall, J M Wardlaw, I R Whittle, Clinical Neurosciences, University of Edinburgh

Competing interests: none declared

\section{REFERENCES}

1 Durcan FJ, Corbett JJ, Wall M. The incidence of pseudotumor cerebri. Population studies in lowa and Louisiana. Arch Neurol 1988;45:875-7

2 Corbett JJ, Savino PJ, Thompson HS, et al. Visual loss in pseudotumor cerebri. Follow-up of 57 patients from five to 41 years and a profile of 14 patients with permanent severe visual loss. Arch Neurol 1982:39:461-74.

3 Sorensen PS, Krogsaa B, Gjerris F. Clinical course and prognosis of pseudotumor cerebri. A prospective study of 24 patients. Acta Neurol Scand 1988;77:164-72.

4 Giuseffi V, Wall M, Siegel PZ, et al. Symptoms and disease associations in idiopathic intracranial hypertension (pseudotumor cerebri): a case-control study. Neurology 1991;41:239-44.
5 Brazis PW, Lee AG. Elevated intracranial pressure and pseudotumor cerebri. Curr Opin Ophthalmol 1998;9:27-32.

6 Walker RW. Idiopathic intracranial hypertension: any light on the mechanism of the raised pressure? J Neurol Neurosurg Psychiatry 2001;71:1-5.

7 Ulug AM, Beauchamp N, Bryan RN, et al. Absolute quantitation of diffusion constants in human stroke. Stroke 1997;28:483-90.

8 Fatouros PP, Marmarou A, Kraft KA, et al. In vivo brain water determination by T1 measurements: effect of total water content, hydration fraction, and field strength. Magn Reson Med 1991;17:402-13.

9 Bastin ME, Sinha S, Whittle IR, et al. Measurements of water diffusion and T1 values in peritumoural oedematous brain. Neuroreport 2002; 13:1335-40.

10 Kingsley PB, Ogg RJ, Reddick WE, et al. Correction of errors caused by imperfect inversion pulses in MR imaging measurement of $\mathrm{T} 1$ relaxation times. Magn Reson Imag 1998; 16:1049-55.

11 Bakker CJG, De Graaf CN, Van Dijk P. Restoration of signal polarity in a set of inversion recovery NMR images. IEEE Trans Med Imag 1984;MI3:197-202.

12 Gideon P, Sorensen PS, Thomsen C, et al. Increased brain water self-diffusion in patients with idiopathic intracranial hypertension. Am J Neuroradiol 1995; 16:381-7.

13 Ulug AM, van Ziil PC. Orientation-independent diffusion imaging without tensor diagonalization: anisotropy definitions based on physical attributes of the diffusion ellipsoid. J Magn Reson Imag 1999;9:804-13.

14 Pierpaoli C, Jezzard P, Basser PJ, et al. Diffusion tensor MR imaging of the human brain. Radiology 1996;201:637-48

15 Sorensen PS, Thomsen C, Gjerris F, et al. Increased brain water content in pseudotumour cerebri measured by magnetic resonance imaging of brain water self diffusion. Neurol Res 1989;11:160-4.

16 Sorensen PS, Thomsen C, Gierris F, et al. Brain water accumulation in pseudotumour cerebri demonstrated by MR-imaging of brain water selfdiffusion. Acta Neurochir Suppl (Wien) 1990;51:363-5.

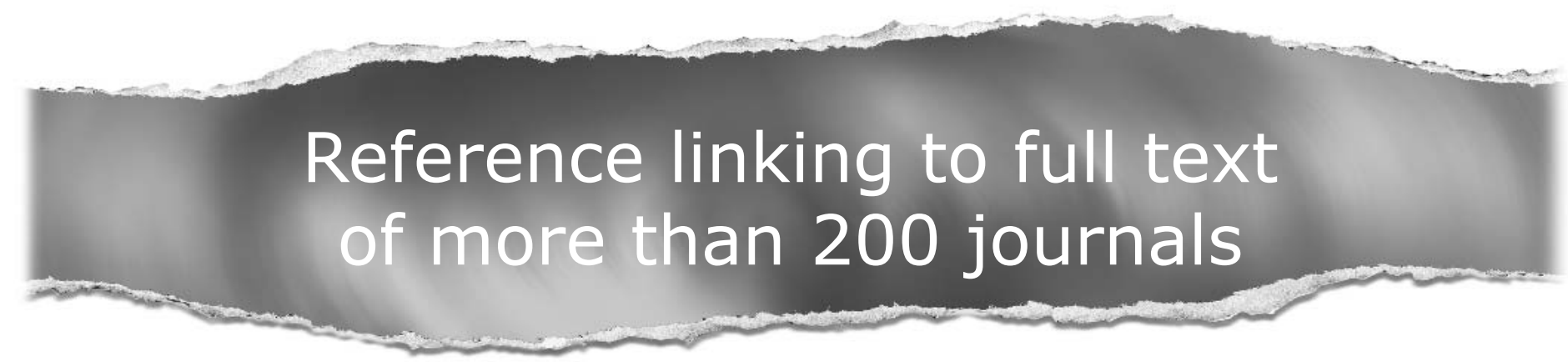

\section{Toll free links}

You can access the FULL TEXT of articles cited in the Journal of Neurology, Neurosurgery, and Psychiatry online if the citation is to one of the more than 200 journals hosted by HighWire (http://highwire.stanford.edu) without a subscription to that journal. There are also direct links from references to the Medline abstract for other titles.

\section{www.jnnp.com}

